\title{
Asymptotic Linearity of Optimal Control Modification Adaptive Law with Analytical Stability Margins
}

\author{
Nhan T. Nguyen* \\ NASA Ames Research Center, Moffett Field, CA 94035
}

\begin{abstract}
Optimal control modification has been developed to improve robustness to model-reference adaptive control. For systems with linear matched uncertainty, optimal control modification adaptive law can be shown by a singular perturbation argument to possess an outer solution that exhibits a linear asymptotic property. Analytical expressions of phase and time delay margins for the outer solution can be obtained. Using the gradient projection operator, a free design parameter of the adaptive law can be selected to satisfy stability margins.
\end{abstract}

\section{Introduction}

Various modifications were developed to increase robustness of MRAC by adding damping to the adaptive law. Two well-known modifications in adaptive control are the $\sigma$-modification ${ }^{1}$ and $\varepsilon$-modification. ${ }^{2}$ These modifications have been used extensively in adaptive control. Recently, a new adaptive law has been introduced that is based on an optimal control formulation to minimize the $\mathscr{L}_{2}$ norm of the tracking error. ${ }^{3}$ The optimality condition results in a damping term proportional to the persistent excitation. The analysis shows that the optimal control modification can allow fast adaptation with a large adaptive gain without causing high-frequency oscillations and can provide improved stability robustness while preserving the tracking performance. This paper shows that this adaptive law can exhibit an asymptotic linear property for systems with linear matched uncertainty using a singular perturbation argument. Due to the asymptotic linear property, the optimal control modification exhibits a linear mapping between the input and output. As a result, it is possible to compute analytically stability margins for this adaptive control scheme. With the use of the gradient projection operator, a free design parameter of the optimal control modification can be designed to satisfy stability margins for its asymptotic solution. Simulation results demonstrate the effectiveness of the method.

\section{Optimal Control Modification Adaptive Law}

Consider a linear plant with uncertainty

$$
\dot{x}=(A+\Delta A) x+(B+\Delta B) u
$$

where $x(t):[0, \infty) \rightarrow \mathbb{R}^{n}$ is a state vector, $u(t):[0, \infty) \rightarrow \mathbb{R}^{p}$ is a control vector, $A \in \mathbb{R}^{n \times n}$ and $B \in \mathbb{R}^{n \times p}, p \geq n$ are constant and known matrices such that the pair $(A, B)$ is controllable, $\Delta A$ and $\Delta B$ are constant but unknown matrices with an assumption that $(A+\Delta A, B+\Delta B)$ is controllable, and furthermore $B+\Delta B=B \Lambda$, where $\Lambda \in \mathbb{R}^{p \times p}$ is an unknown control effectiveness diagonal matrix with positive elements.

Assumption 1: $\Delta A$ is a matched uncertainty whereby there exists a contant matrix $\Omega \in \mathbb{R}^{n \times p}$ such that $\Delta A=$ $(B+\Delta B) \Omega^{\top}$. Moreover, if $p \geq n$ and if $\operatorname{rank}\left((B+\Delta B)(B+\Delta B)^{\top}\right)=n$, then $\Delta A$ exists and

$$
\Omega^{\top}=(B+\Delta B)^{\top}\left[(B+\Delta B)(B+\Delta B)^{\top}\right]^{-1} \Delta A
$$

A nominal fixed gain controller has been designed to stabilize the plant and enable it to track a command $r(t)$

$$
u_{\text {nom }}=-K_{x} x-K_{r} r
$$

${ }^{*}$ Research Scientist, Intelligent Systems Division, Mail Stop 269-1, AIAA Associate Fellow 
where $r(t):[0, \infty) \rightarrow \mathbb{R}^{r} \in \mathscr{L}_{\infty}$ is a piecewise-continuous and bounded command vector, $A-B K_{x} \in \mathbb{R}^{n \times n}$ is Hurwitz, and $B K_{r} \in \mathbb{R}^{n \times r}$ with $r \leq n$.

The closed-loop nominal plant without uncertainty is

$$
\dot{x}=A_{m} x+B_{m} r
$$

This closed-loop nominal plant is then used to specify a reference model

$$
\dot{x}_{m}=A_{m} x_{m}+B_{m} r
$$

where $x_{m}(t):[0, \infty) \rightarrow \mathbb{R}^{n}$ is a reference state vector, and $A_{m}=A-B K_{x}$ and $B_{m}=-B K_{r}$.

Since $r(t)$ is bounded, then $x_{m}(t)$ can be shown to be uniformly bounded such that

$$
\left\|x_{m}(0)\right\|<\varepsilon \Rightarrow\left\|x_{m}(t)\right\| \leq \delta(\varepsilon), \forall t \geq 0
$$

The objective is to design a full-state feedback adaptive augmentation controller to enable $x(t)$ to follow $x_{m}(t)$ in the presence of uncertainty due to $\Delta A$ and $\Delta B$ with the following controller

$$
u=u_{\text {nom }}+u_{a d}
$$

where $u_{a d}(t):[0, \infty) \rightarrow \mathbb{R}^{p}$ is an adaptive augmentation controller.

Assumption 2: There exist ideal constant matrices $\Theta_{x}^{*}$ and $\Theta_{r}^{*}$ such that the following conditions are satisfied

$$
\begin{gathered}
\Delta A-\Delta B K_{x}=(B+\Delta B) \Theta_{x}^{* \top} \\
-\Delta B K_{r}=(B+\Delta B) \Theta_{r}^{* \top}
\end{gathered}
$$

Let $\Theta^{* \top}=\left[\begin{array}{cc}\Theta_{x}^{* \top} & \Theta_{r}^{* \top}\end{array}\right]: \mathbb{R}^{p \times n} \times \mathbb{R}^{p \times r} \rightarrow \mathbb{R}^{p \times(m=n+r)}$ and $\Phi(x, r)=\left[\begin{array}{cc}x^{\top}(t) & r^{\top}(t)\end{array}\right]^{\top}: \mathbb{R}^{n} \times \mathbb{R}^{r} \rightarrow \mathbb{R}^{m}$. Define a predictor model of the uncertain plant as

$$
\dot{\hat{x}}=A_{m} x+B_{m} r+(B+\Delta B) \Theta^{* \top} \Phi+(B+\Delta \hat{B}) u_{a d}
$$

where $\dot{\hat{x}}(t):[0, \infty) \rightarrow \mathbb{R}^{n}$ is an estimate of $\dot{x}(t)$ and $\Delta \hat{B}(t):[0, \infty) \rightarrow \mathbb{R}^{n \times p}$ is an estimate of $\Delta B$.

Choose an adaptive controller of the form

$$
u_{a d}=-\Theta^{\top} \Phi
$$

Then the closed-loop predictor model is described by

$$
\dot{\hat{x}}=A_{m} x+B_{m} r-(B+\Delta \hat{B}) \tilde{\Theta}^{\top} \Phi-\Delta \tilde{B} \Theta^{\top} \Phi+\Delta \tilde{B} \tilde{\Theta}^{\top} \Phi
$$

where $\Delta \tilde{B}(t)=\Delta \hat{B}(t)-\Delta B$ and $\tilde{\Theta}(t)=\Theta(t)-\Theta^{*}$ are estimation errors.

Define the tracking error dynamics as $\dot{e}(t)=\dot{x}_{m}(t)-\dot{\hat{x}}(t)$, then the tracking error equation becomes

$$
\dot{e}=A_{m} e+\hat{B} \tilde{\Theta}^{\top} \Phi+\Delta \tilde{B} \Theta^{\top} \Phi+\varepsilon
$$

where $\hat{B}(t)=B+\Delta \hat{B}(t)$ and $\varepsilon(x, r):: \mathbb{R}^{n} \times \mathbb{R}^{r} \rightarrow \mathbb{R}^{n}$ is a second-order predictor error

$$
\varepsilon=-\Delta \tilde{B} \tilde{\Theta}^{\top} \Phi
$$

Proposition 1: The following adaptive laws

$$
\begin{gathered}
\dot{\Theta}=-\Gamma_{\Theta} \Phi\left(e^{\top} P-v \Phi^{\top} \Theta \hat{B}^{\top} P A_{m}^{-1}\right) \hat{B} \\
\Delta \dot{\hat{B}}^{\top}=-\Gamma_{B} \Theta^{\top} \Phi\left(e^{\top} P-v \Phi^{\top} \Theta \hat{B}^{\top} P A_{m}^{-1}\right)
\end{gathered}
$$

is a solution that minimizes the following an infinite-time horizon cost function

$$
J=\lim _{t_{f} \rightarrow \rightarrow \infty} \frac{1}{2} \int_{0}^{t_{f}}(e-\Delta)^{\top} Q(e-\Delta) d t
$$


where $\Delta(t):[0, \infty) \rightarrow \mathbb{R}^{n}$ is a lower bound of the tracking error, $\Gamma_{\Theta}=\Gamma_{\Theta}^{\top}>0 \in \mathbb{R}^{m \times m}$ and $\Gamma_{B}=\Gamma_{B}^{\top}>0 \in \mathbb{R}^{p \times p}$ are adaptive gain matrices, $v>0 \in \mathbb{R}$ is a free design parameter, and $P=P^{\top}>0 \in \mathbb{R}^{n \times n}$ solves

$$
P A_{m}+A_{m}^{\top} P=-Q
$$

where $Q=Q^{\top}>0 \in \mathbb{R}^{n \times n}$.

Proof: The cost function $J$ is convex and represents the distance measured from a point on the trajectory of $e(t)$ to the normal surface of a hypersphere $\Omega=\left\{e(t) \in \mathbb{R}^{n}:\|e\| \leq\|\Delta\|\right\} \subset \mathscr{D} \subset \mathbb{R}^{n}$. The cost function is designed to provide robustness by not seeking an asymptotic tracking error that tends to zero but rather a tracking error that tends to some lower bound away from the origin. By not requiring $e(t) \rightarrow 0$ as $t \rightarrow \infty$, the adaptation can be made to be robust. Therefore, the tracking performance can be traded with robustness by a suitable selection of the tuning parameter $v$.

An optimal control problem can be formulated by the Pontryagin's Minimum Principle. Define a Hamiltonian

$$
H=\frac{1}{2}(e-\Delta)^{\top} Q(e-\Delta)+p^{\top}\left(A_{m} e+\hat{B} \tilde{\Theta}^{\top} \Phi+\Delta \tilde{B} \Theta^{\top} \Phi+\varepsilon\right)
$$

where $p(t):[0, \infty) \rightarrow \mathbb{R}^{n}$ is an adjoint variable, then the necessary condition can be established by

$$
\dot{p}=-\nabla H_{e}^{\top}=-Q(e-\Delta)-A_{m}^{\top} p
$$

with the transversality condition $p\left(t_{f} \rightarrow \infty\right)=0$ since $e(0)$ is known.

Then the adaptive laws can be formulated by a gradient method as ${ }^{4}$

$$
\begin{gathered}
\dot{\tilde{\Theta}}=-\Gamma_{\Theta} \nabla H_{\tilde{\Theta}^{\top}}=-\Gamma_{\Theta} \Phi p^{\top} \hat{B} \\
\Delta \dot{\tilde{B}}^{\top}=-\Gamma_{B} \nabla H_{\Delta \tilde{B}}=-\Gamma_{B} \Theta^{\top} \Phi p^{\top}
\end{gathered}
$$

An "approximate" solution of $p(t)$ can be obtained using a "sweeping" method ${ }^{5}$ by letting $p=P e+S \hat{B} \Theta^{\top} \Phi$. Then

$$
\dot{P} e+P\left[A_{m} e+\hat{B}\left(\Theta^{\top}-\Theta^{* \top}\right) \Phi+\Delta \tilde{B} \Theta^{\top} \Phi+\varepsilon\right]+\dot{S} \hat{B} \Theta^{\top} \Phi+S \frac{d\left(\hat{B} \Theta^{\top} \Phi\right)}{d t}=-Q(e-\Delta)-A_{m}^{\top}\left(P e+S \hat{B} \Theta^{\top} \Phi\right)
$$

which yields the following equations

$$
\begin{gathered}
\dot{P}+P A_{m}+A_{m}^{\top} P+Q=0 \\
\dot{S}+P+A_{m}^{\top} S=0
\end{gathered}
$$

subject to the transversality conditions $P\left(t_{f} \rightarrow \infty\right)=0$ and $S\left(t_{f} \rightarrow \infty\right)=0$, ans

$$
Q \Delta=S \frac{d\left(\hat{B} \Theta^{\top} \Phi\right)}{d t}-P(B+\Delta B) \Theta^{* \top} \Phi
$$

Introducing a time-to-go variable $\tau=t_{f}-t$, then the Lyapunov differential equation (24) becomes

$$
-\frac{d P}{d \tau}+P A_{m}+A_{m}^{\top} P+Q=0
$$

subject to the initial conditions in time-to-go $P(0)=0$, for which the existence and uniqueness of its solution is well-established.

. It follows that Eq. (25) also has a stable, unique solution in time-to-go since $A_{m}$ is Hurwitz

$$
-\frac{d S}{d \tau}+P B+A_{m}^{\top} S=0
$$

subject to $S(0)=0$.

The solutions of $P(\tau)$ and $S(\tau)$ as $t_{f} \rightarrow \infty$ approach their steady-state constant solutions

$$
\begin{gathered}
P A_{m}+A_{m}^{\top} P=-Q \\
S=-A_{m}^{-\top} P
\end{gathered}
$$


Without any loss of generality, a free design parameter $v>0 \in \mathbb{R}$ is introduced as a gain to allow for adjustments of the modification term in the adaptive law, where $v=1$ corresponds to an optimal solution. Thus

$$
S=-v A_{m}^{-\top} P
$$

The adjoint $p$ is then obtained as

$$
p=P e-v A_{m}^{-\top} P \hat{B} \Theta^{\top} \Phi
$$

Substituting $p$ into the gradient adaptive laws yields

$$
\begin{gathered}
\dot{\Theta}=-\Gamma_{\Theta} \Phi\left(e^{\top} P-v \Phi^{\top} \Theta \hat{B}^{\top} P A_{m}^{-1}\right) \hat{B} \\
\Delta \dot{\hat{B}}^{\top}=-\Gamma_{B} \Theta^{\top} \Phi\left(e^{\top} P-v \Phi^{\top} \Theta \hat{B}^{\top} P A_{m}^{-1}\right)
\end{gathered}
$$

Theorem 1: The adaptive laws (15) and (16) result in stable and uniformly ultimately bounded tracking error $e(t)$ for all $(e(0), \tilde{\Theta}(0), \Delta \tilde{B}(0)) \in B_{\alpha}$ with an ultimate bound

$$
\rho=\sqrt{\frac{\lambda_{\max }(P) r^{2}+\lambda_{\max }\left(\Gamma_{\Theta}^{-1}\right) \kappa^{2}+\lambda_{\max }\left(\Gamma_{B}^{-1}\right) v^{2}}{\lambda_{\min }(P)}}
$$

where

$$
\begin{gathered}
r=\frac{2\|P\| \varepsilon_{0}}{\lambda_{\min }(Q)} \\
\kappa=\frac{2\left\|P A_{m}^{-1}\right\| \Theta_{0}}{\lambda_{\min }\left(A_{m}^{-\top} Q A_{m}^{-1}\right)} \\
v=\frac{2\left\|P A_{m}^{-1}\right\| \Delta B_{0}}{\lambda_{\text {min }}\left(A_{m}^{-\top} Q A_{m}^{-1}\right)}
\end{gathered}
$$

with $\varepsilon_{0}=\sup _{x \in \mathscr{D}, r \in \mathbb{R}^{r}}\|\varepsilon(x, r)\|, \Theta_{0}=\left\|\Theta^{*}\right\|$, and $\Delta B_{0}=\|\Delta B\|$.

Proof: Choose a Lyapunov candidate function

$$
V=e^{\top} P e+\operatorname{trace}\left(\tilde{\Theta}^{\top} \Gamma_{\Theta}^{-1} \tilde{\Theta}\right)+\operatorname{trace}\left(\Delta \tilde{B} \Gamma_{B}^{-1} \Delta \tilde{B}^{\top}\right)
$$

Evaluating $\dot{V}$ yields

$$
\begin{array}{r}
\dot{V}=e^{\top}\left(A_{m} P+P A_{m}\right) e+2 e^{\top} P\left(\hat{B} \tilde{\Theta}^{\top} \Phi+\Delta \tilde{B} \Theta^{\top} \Phi+\varepsilon\right)-2 \operatorname{trace}\left[\tilde{\Theta}^{\top} \Phi\left(e^{\top} P-v \Phi^{\top} \Theta \hat{B}^{\top} P A_{m}^{-1}\right) \hat{B}\right] \\
-2 \operatorname{trace}\left[\Delta \tilde{B} \Theta^{\top} \Phi\left(e^{\top} P-v \Phi^{\top} \Theta \hat{B}^{\top} P A_{m}^{-1}\right)\right]
\end{array}
$$

Using the trace identity trace $\left(A^{\top} B\right)=B A^{\top}, \dot{V}$ can be written as

$$
\begin{aligned}
\dot{V}=-e^{\top} Q e+2 e^{\top} P\left(\hat{B} \tilde{\Theta}^{\top} \Phi+\Delta \tilde{B} \Theta^{\top} \Phi+\varepsilon\right)-2 e^{\top} P \hat{B} \tilde{\Theta}^{\top} \Phi \\
+2 v \Phi^{\top} \Theta \hat{B}^{\top} P A_{m}^{-1} \hat{B} \tilde{\Theta}^{\top} \Phi-2 e^{\top} P \Delta \tilde{B} \Theta^{\top} \Phi+2 \nu \Phi^{\top} \Theta \hat{B}^{\top} P A_{m}^{-1} \Delta \tilde{B} \Theta^{\top} \Phi
\end{aligned}
$$

The sign-definiteness of the term $P A_{m}^{-1}$ is now considered. Recall that a general real matrix $G$ is positive (negative) definite if and only if its symmetric part $M=\frac{1}{2}\left(G+G^{\top}\right)$ is also positive (negative) definite. Then, by pre- and postmultiplication of Eq. (18) by $A_{m}^{-\top}$ and $A_{m}^{-1}$, respectively, $P A_{m}^{-1}$ can be decomposed into a symmetric part $M$ and anti-symmetric part $N$ as

$$
P A_{m}^{-1}=M+N
$$

where

$$
\begin{gathered}
M=\frac{1}{2}\left(A_{m}^{-\top} P+P A_{m}^{-1}\right)=-\frac{1}{2} A_{m}^{-\top} Q A_{m}^{-1} \\
N=\frac{1}{2}\left(P A_{m}^{-1}-A_{m}^{-\top} P\right) .
\end{gathered}
$$


Since the symmetric part $M<0$, then $P A_{m}^{-1}<0$. Thus, $\dot{V}$ becomes

$$
\dot{V}=-e^{\top} Q e+2 e^{\top} P \varepsilon+2 v \Phi^{\top}\left(\Theta^{*}+\tilde{\Theta}\right) \hat{B}^{\top}(M+N) \hat{B} \tilde{\Theta}^{\top} \Phi+2 v \Phi^{\top} \Theta(\Delta B+\Delta \tilde{B})^{\top}(M+N) \Delta \tilde{B} \Theta^{\top} \Phi
$$

Using the property $y^{\top} N y=0$ for an anti-symmetric matrix $N, \dot{V}$ is reduced to

$$
\begin{aligned}
\dot{V}=-e^{\top} Q e+2 e^{\top} P \varepsilon+2 v \Phi^{\top} \Theta^{*} \hat{B}^{\top} P A_{m}^{-1} \hat{B} \tilde{\Theta}^{\top} \Phi-v \Phi^{\top} \tilde{\Theta} \hat{B}^{\top} A_{m}^{-\top} Q A_{m}^{-1} \hat{B} \tilde{\Theta}^{\top} \Phi \\
\\
+2 v \Phi^{\top} \Theta \Delta B^{\top} P A_{m}^{-1} \Delta \tilde{B} \Theta^{\top} \Phi-v \Phi^{\top} \Theta \Delta \tilde{B}^{\top} A_{m}^{-\top} Q A_{m}^{-1} \Delta \tilde{B} \Theta^{\top} \Phi
\end{aligned}
$$

which is bounded by

$$
\begin{array}{r}
\dot{V} \leq-\|e\|\left[\lambda_{\min }(Q)\|e\|-2\|P\| \varepsilon_{0}\right]-v\|\hat{B}\|^{2}\|\Phi\|^{2}\|\tilde{\Theta}\|\left[\lambda_{\min }\left(A_{m}^{-\top} Q A_{m}^{-1}\right)\|\tilde{\Theta}\|-2\left\|P A_{m}^{-1}\right\| \Theta_{0}\right] \\
-v\|\Theta\|^{2}\|\Phi\|^{2}\|\Delta \tilde{B}\|\left[\lambda_{\min }\left(A_{m}^{-\top} Q A_{m}^{-1}\right)\|\Delta \tilde{B}\|-2\left\|P A_{m}^{-1}\right\| \Delta B_{0}\right]
\end{array}
$$

Let

$$
B_{r}=\left\{(e, \tilde{\Theta}, \Delta \tilde{B}) \in \mathbb{R}^{n} \times \mathbb{R}^{m \times p} \times \mathbb{R}^{n \times p}:\|e\| \leq r \text { or }\|\tilde{\Theta}\| \leq \kappa \text { or }\|\Delta \tilde{B}\| \leq v\right\}
$$

where

$$
\begin{gathered}
\|e\| \leq r=\frac{2\|P\| \varepsilon_{0}}{\lambda_{\min }(Q)} \\
\|\tilde{\Theta}\| \leq \kappa=\frac{2\left\|P A_{m}^{-1}\right\| \Theta_{0}}{\lambda_{\min }\left(A_{m}^{-\top} Q A_{m}^{-1}\right)} \\
\|\Delta \tilde{B}\| \leq v=\frac{2\left\|P A_{m}^{-1}\right\| \Delta B_{0}}{\lambda_{\text {min }}\left(B^{\top} A_{m}^{-\top} Q A_{m}^{-1} B\right)}
\end{gathered}
$$

It follows that $\dot{V} \leq 0$ for all $(e, \tilde{\Theta}, \Delta \tilde{B}) \in B_{R}-B_{r}$, where $B_{R}=\left\{e \in \mathbb{R}^{n}:\|e\| \leq R\right\} \subset \mathscr{D}$. Let $B_{\beta}$ be the smallest subset that encloses $B_{r}$, then there exists $\beta>0$ where

$$
\beta=\lambda_{\max }(P) r^{2}+\lambda_{\max }\left(\Gamma_{\Theta}^{-1}\right) \kappa^{2}+\lambda_{\max }\left(\Gamma_{B}^{-1}\right) v^{2}
$$

such that

$$
B_{r} \subset B_{\beta}=\left\{(e, \tilde{\Theta}, \Delta \tilde{B}) \in \mathbb{R}^{n} \times \mathbb{R}^{m \times p} \times \mathbb{R}^{n \times p}: V \leq \beta\right\}
$$

Let $B_{\alpha}$ be the largest subset enclosed by $B_{R}$, then since $\|e\| \leq R$ in $B_{R}$, there exists $\alpha>0$ where

$$
\lambda_{\text {min }}(P)\|e\|^{2} \leq \lambda_{\text {min }}(P)\|e\|^{2}+\lambda_{\text {min }}\left(\Gamma_{\Theta}^{-1}\right)\|\tilde{\Theta}\|^{2}+\lambda_{\text {min }}\left(\Gamma_{B}^{-1}\right)\|\Delta \tilde{B}\|^{2} \leq V \leq \lambda_{\text {min }}(P) R^{2}=\alpha
$$

such that

$$
B_{\alpha}=\left\{(e, \tilde{\Theta}, \Delta \tilde{B}) \in \mathbb{R}^{n} \times \mathbb{R}^{m \times p} \times \mathbb{R}^{n \times p}: V \leq \alpha\right\} \subset B_{R}
$$

Then for a solution to be uniformly bounded, the set containment is as follows:

$$
B_{r} \subset B_{\beta} \subset B_{\alpha} \subset B_{R}
$$

This implies

$$
\beta<\alpha \Leftrightarrow \lambda_{\max }(P) r^{2}+\lambda_{\max }\left(\Gamma_{\Theta}^{-1}\right) \kappa^{2}+\lambda_{\max }\left(\Gamma_{B}^{-1}\right) v^{2}<\lambda_{\min }(P) R^{2}
$$

Therefore

$$
R>\sqrt{\frac{\lambda_{\max }(P) r^{2}+\lambda_{\max }\left(\Gamma_{\Theta}^{-1}\right) \kappa^{2}+\lambda_{\max }\left(\Gamma_{B}^{-1}\right) v^{2}}{\lambda_{\min }(P)}}=\rho
$$

where $\rho$ is the smallest value of $R$.

Then $\rho$ is the ultimate bound of $e(t)$ such that

$$
r \leq\|e\| \leq \rho \leq R
$$

Since $\dot{V} \leq 0$ for all $(e, \tilde{\Theta}, \Delta \tilde{B}) \in B_{R}-B_{r}$, therefore $V$ is a decreasing function of time outside of $B_{r}$. Thus, if $(e(0), \tilde{\Theta}(0), \Delta \tilde{B}(0)) \in B_{\alpha}$, then according to Theorem 5.1 of Ref., ${ }^{6}$ the solution will eventually enters $B_{\beta}$ after a finite time $t=T$ (independent of $(e(0), \tilde{\Theta}(0), \Delta \tilde{B}(0))$ and $\alpha$ ) and remain inside for all $t>T$. Therefore, $e(t)$ is uniformly ultimately bounded with an ultimate bound $\rho$. 


\section{Asymptotic Linearity}

Note that the tracking error equation can also be written as

$$
\dot{e}=A_{m} e-(B+\Delta B) \Theta^{* \top} \Phi+\hat{B} \Theta^{\top} \Phi
$$

Now consider a time-scale separation problem when $\left\|\Gamma_{\Theta}\right\|=\frac{\gamma_{\Theta}}{\varepsilon} \gg\left\|A_{m}\right\|$ and $\left\|\Gamma_{B}\right\|=\frac{\gamma_{B}}{\varepsilon} \gg\left\|A_{m}\right\|$ where $\varepsilon$ is a small parameter. Then $\Theta(t)$ and $\Delta \hat{B}(t)$ are said to be a fast state and $e(t)$ is a slow state. To decouple the fast and slow states, a time-scale separation can be performed by applying the singular perturbation method using a time transformation

$$
\tau=\varepsilon t
$$

where $\tau$ is a slow time variable.

Then, the system is transformed into a singularly perturbed system as

$$
\begin{gathered}
\frac{d e}{d \tau}=\frac{1}{\varepsilon}\left[A_{m} e-(B+\Delta B) \Theta^{* \top} \Phi+\hat{B} \Theta^{\top} \Phi\right] \\
\varepsilon \frac{d \Theta}{d \tau} \approx-\frac{\gamma_{\Theta}}{\varepsilon} \Phi\left(e^{\top} P-v \Phi^{\top} \Theta \hat{B}^{\top} P A_{m}^{-1}\right) \hat{B} \\
\varepsilon \frac{d \Delta \hat{B}^{\top}}{d \tau} \approx-\frac{\gamma_{B}}{\varepsilon} \Theta^{\top} \Phi\left(e^{\top} P-v \Phi^{\top} \Theta \hat{B}^{\top} P A_{m}^{-1}\right)
\end{gathered}
$$

The Tikhonov's theorem can be used to approximate the solution of the singularly perturbed system with the solution of a "reduced-order" system by letting $\varepsilon \rightarrow 0 .^{7}$ Then, $\Theta(e, \varepsilon)$ and $\Delta \hat{B}$ are on a fast manifold. Thus, the reduced-order system is given by

$$
\begin{gathered}
\varepsilon^{2} \frac{d \Theta}{d \tau} \approx-\gamma_{\Theta} \Phi\left(e^{\top} P-v \Phi^{\top} \Theta \hat{B}^{\top} P A_{m}^{-1}\right) \hat{B} \Rightarrow\left(\hat{B} \Theta^{\top} \Phi\right)^{(0)}=\frac{1}{v} P^{-1} A_{m}^{-1} P e^{(0)} \\
\dot{e}^{(0)}=A_{m} e^{(0)}-(B+\Delta B) \Theta^{* \top} \Phi^{(0)}+\left(\hat{B} \Theta^{\top} \Phi\right)^{(0)}
\end{gathered}
$$

where $e^{(0)}(t)$ and $\left(\hat{B} \Theta^{\top} \Phi\right)^{(0)}(t)$ are the "outer" solutions of the singularly perturbed system.

The term "outer" is in connection with the concept of "inner" or "boundary layer" and "outer" solutions which have the origin in boundary layer theory due to Prandtl. The "inner" or "boundary layer" solution for this system is obtained from

$$
\begin{gathered}
\dot{\Theta}^{(i)}=-\Gamma_{\Theta} \Phi^{(i)}\left(e^{(i) \top} P-v\left(\Phi^{\top} \Theta \hat{B}^{\top}\right)^{(i)} P A_{m}^{-1}\right) \hat{B}^{(i)} \\
\Delta \dot{\hat{B}}^{\top}=-\Gamma_{B} \Theta^{(i) \top} \Phi^{(i)}\left(e^{(i) \top} P-v\left(\Phi^{\top} \Theta \hat{B}^{\top}\right)^{(i)} P A_{m}^{-1}\right) \\
\varepsilon \frac{d e}{d \tau}=A_{m} e-(B+\Delta B) \Theta^{* \top} \Phi+\hat{B} \Theta \Phi \Rightarrow A_{m} e^{(i)}=(B+\Delta B) \Theta^{* \top} \Phi^{(i)}-\left(\hat{B} \Theta^{\top} \Phi\right)^{(i)}
\end{gathered}
$$

The general solution of the system is then expressed as

$$
e(t)=e^{(0)}(t)+e^{(i)}(t)-e_{M A E}(t)
$$

where $e_{M A E}(t)$ is a correction term by a matched asymptotic expansion method applied to both the inner and outer solutions. ${ }^{8}$ The outer solution is in fact the asymptotic solution of the original system as $t \rightarrow \infty$. For the singularly perturbed system comprising $e^{(0)}(t)$ and $\left(\hat{B} \Theta^{\top} \Phi\right)^{(0)}(t)$, the outer solution of the tracking error is then determined by

$$
\dot{e}^{(0)}=\left[A_{m}+\frac{1}{v} P^{-1} A_{m}^{\top} P+\left(\Delta A-\Delta B K_{x}\right)\right] e^{(0)}-\left(\Delta A-\Delta B K_{x}\right) x_{m}+\Delta B K_{r} r
$$

which interestingly enough is an LTI equation.

Since the asymptotic tracking error equation for the outer solution is LTI, many standard LTI analysis tools can be used to estimate the asymptotic behavior of the original system. In particular, the free design parameter $v$ can be selected to provide a notion of stability margin for the outer solution. 
The projection operator can be applied to the adaptive laws with a priori knowledge of the bounds on $\Delta A$ and $\Delta B$. Using these a priori bounds, the free design parameter $v$ can be selected to guarantee stability robustness. Then the adaptive laws are modified by the projection operator as follows:

$$
\begin{gathered}
\dot{\Theta}=-\Gamma_{\Theta} \operatorname{Proj}\left(\Theta, \Phi\left(e^{\top} P-v \Phi^{\top} \Theta \hat{B}^{\top} P A_{m}^{-1}\right) \hat{B}\right) \\
\Delta \dot{\hat{B}}^{\top}=-\Gamma_{B} \operatorname{Proj}\left(\Delta \hat{B}, \Theta^{\top} \Phi\left(e^{\top} P-v \Phi^{\top} \Theta \hat{B}^{\top} P A_{m}^{-1}\right)\right)
\end{gathered}
$$

The free design parameter $v$ can now be estimated to give the asymptotic closed-loop tracking error a desired measure of stability robustness such as phase or time delay margin. One method for estimating a MIMO phase or time delay margin is based on a matrix measure $\operatorname{method}^{9}$ which is as follows

In the presence of an input delay, the closed-loop tracking error becomes

$$
\dot{e}^{(0)}(t)=(A+\Delta A) e^{(0)}(t)-\left[(B+\Delta B) K_{x}-\frac{1}{v} P^{-1} A_{m}^{\top} P\right] e^{(0)}\left(t-t_{d}\right)-\left(\Delta A-\Delta B K_{x}\right) x_{m}\left(t-t_{d}\right)+\Delta B K_{r} r\left(t-t_{d}\right)
$$

The MIMO phase and time margins can be estimated by

$$
\begin{gathered}
\phi=\cos ^{-1} \frac{\bar{\mu}(A+\Delta A)+\bar{\mu}\left(j\left[(B+\Delta B) K_{x}-\frac{1}{v} P^{-1} A_{m}^{\top} P\right]\right)}{\left\|(B+\Delta B) K_{x}-\frac{1}{v} P^{-1} A_{m}^{\top} P\right\|} \\
t_{d m}=\frac{\phi}{\bar{\mu}(-j A-j \Delta A)+\left\|(B+\Delta B) K_{x}-\frac{1}{v} P^{-1} A_{m}^{\top} P\right\|}
\end{gathered}
$$

where $\bar{\mu}$ as the maximum eigenvalue of a symmetric part of a general complex matrix $C$

$$
\bar{\mu}(C)=\lambda_{\max }\left(\frac{C+C^{*}}{2}\right)
$$

where $C^{*}$ is the complex conjugate of $C$.

Utilizing this equation, the free design parameter $v$ can then be estimated to provide a measure of a phase margin in an asymptotic sense.

Example: Instability of adaptive systems with unmodeled dynamics is a well-known phenomenon. ${ }^{10}$ Consider a stable first-order plant coupled with a second-order unmodeled plant with two highly damped poles which could represent a structural mode

$$
\begin{gathered}
\dot{x}=-x+2 u-0.1 y \\
\ddot{y}+2 \zeta \omega_{n} \dot{y}+\omega_{n} y=7 x
\end{gathered}
$$

where $\zeta=5$ and $\omega_{n}=10$.

The reference model is

$$
\dot{x}_{m}=-2 x_{m}+2 r
$$

where the reference command is a step input with a small sinusoidal variation at the same frequency as $\omega_{n}$ and a small noise signal

$$
r=1+0.1 \sin \omega_{n} t
$$

The controller is given by

$$
u=\Theta^{\top} \Phi
$$

where $\Phi(t)=\left[\begin{array}{ll}x(t) & r(t)\end{array}\right]^{\top}$ and $\Theta(t)=\left[\begin{array}{ll}\theta_{x}(t) & \theta_{r}(t)\end{array}\right]^{\top}$ is updated by

$$
\dot{\Theta}(t)=\Gamma\left(\Phi e-v \Phi \Phi^{\top} \Theta\right)
$$

with $e(t)=x(t)-x_{m}(t), \Theta(0)=0$, and $\Gamma=I$.

The conventional MRAC, i.e., $v=0$, leads to an instability as shown in Fig. 1 even though the open-loop plant is stable. A value of $v=80$ is chosen, the optimal control modification is able to produce a stabilizing controller, as seen in Fig. 2. Thus, this illustrates the robustness of the optimal control modification. As a comparison, the $\varepsilon$-modification is used instead for the update law

$$
\dot{\Theta}=\Gamma(\Phi e-\mu|e| \Theta)
$$


with $\mu=80$. The adaptive signal is stable as shown in Fig. 3 .

The asymptotic tracking error equation for fast adaptation is

$$
u \rightarrow \frac{e}{v}
$$

The asymptotic closed-loop plant can be expressed as

$$
\left(s+1-\frac{2}{v}+\frac{0.7}{s^{2}+2 \zeta \omega_{n} s+\omega_{n}^{2}}\right) x=-\frac{4 r}{v(s+2)}
$$

As $t \rightarrow \infty$

$$
\frac{x}{r} \rightarrow-\frac{2 \omega_{n}^{2}}{(v-2) \omega_{n}^{2}+0.7 v}
$$

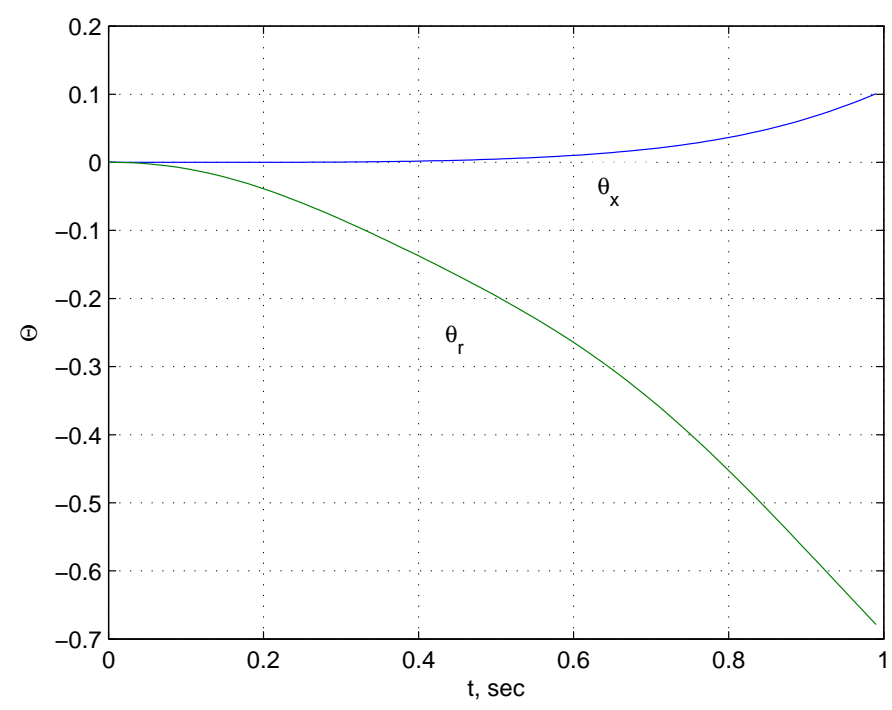

Fig. 1 - Instability due to MRAC, $v=0$

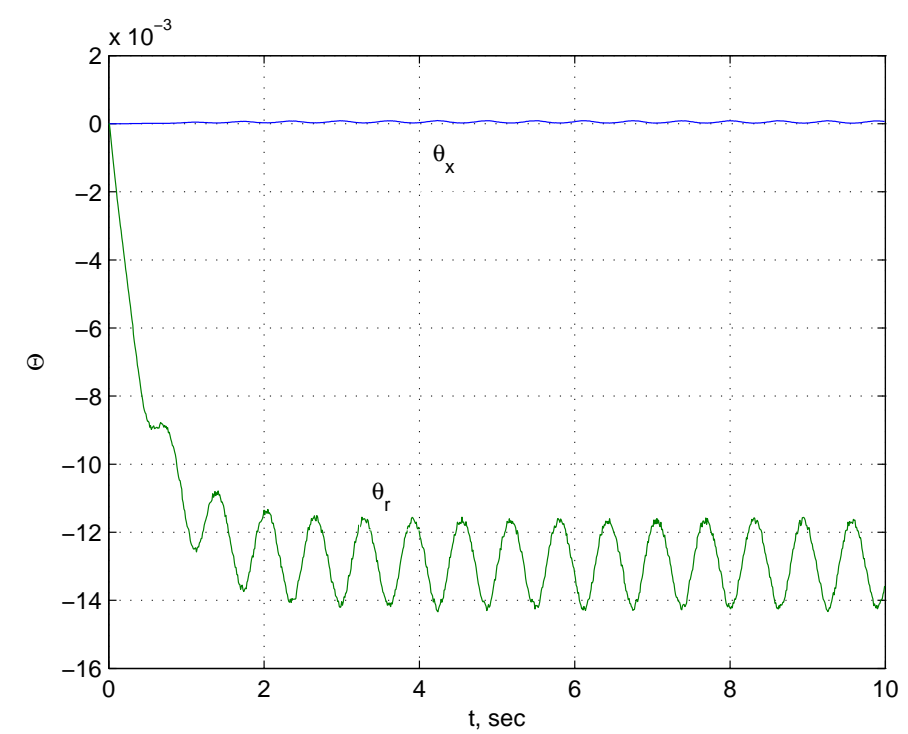

Fig. 2 - Stable Adaptation with Optimal Control Modification, $v=80$ 


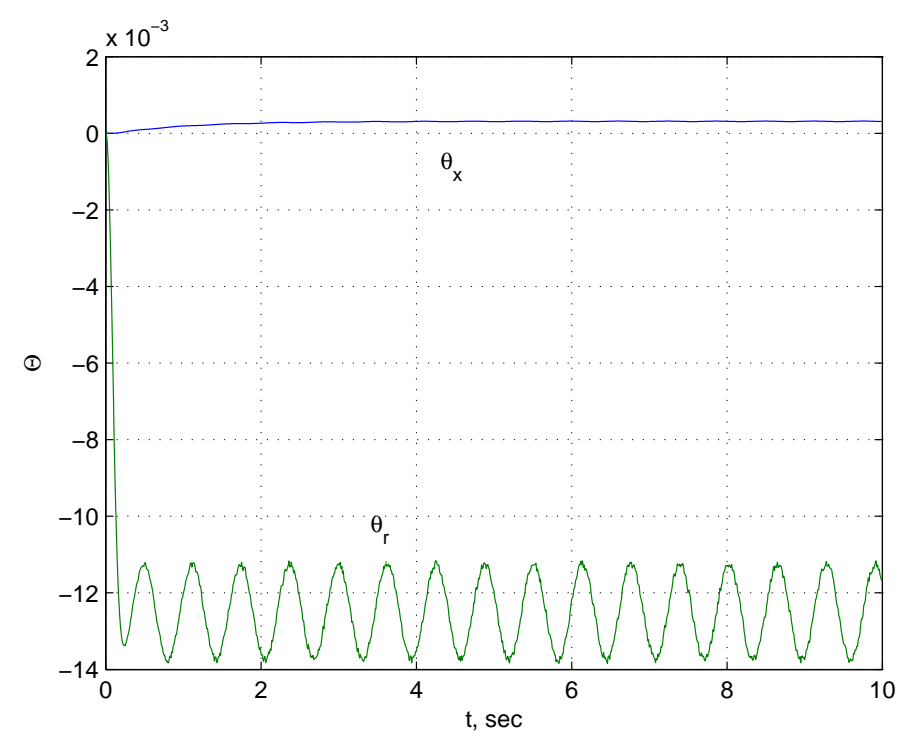

Fig. 3 - Stable Adaptation with $\varepsilon$-Modification, $\mu=80$

Figure 4 illustrates the linear mapping between $x$ and $r$ for three different multipliers $0.1,0.5$, and 1.0 that scale the input signal. The predicted asymptotic ratio of $x$ to $r$ is -0.0255 , which agrees surprising well with the simulation results. In contrast, Fig. 5 shows that the $\varepsilon$-modification results in a nonlinear mapping between $x$ and $r$.

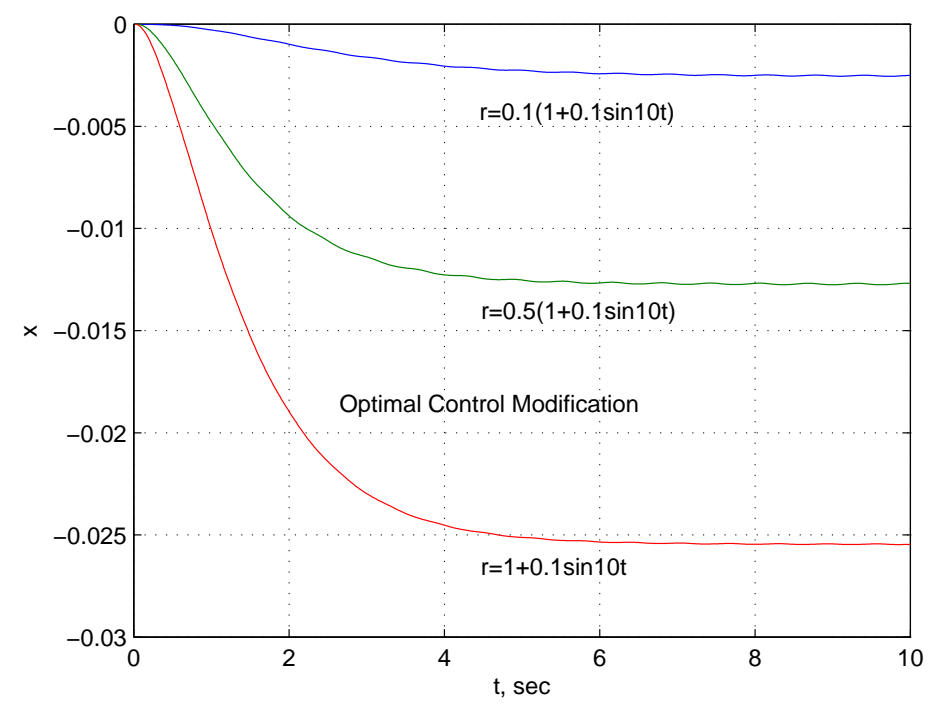

Fig. 4 - Input-Output Linear Mapping by Optimal Control Modification 


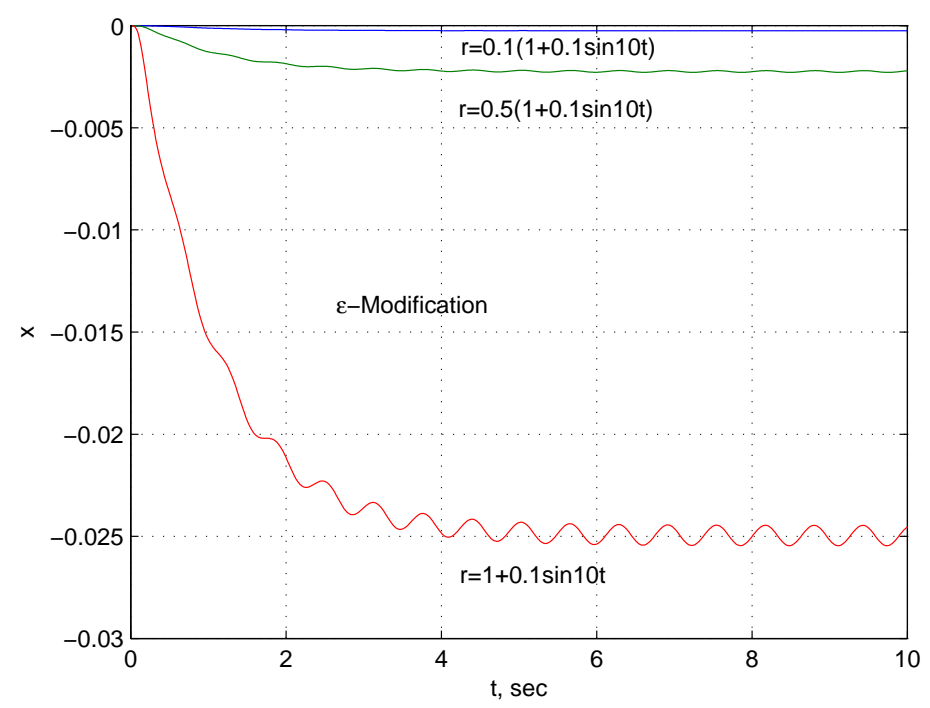

Fig. 5 - Input-Output Nonlinear Mapping by $\varepsilon$ - Modification

\section{Flight Control Simulation}

Consider a longitudinal pitch dynamical model of an aircraft

$$
\left[\begin{array}{ccc}
m V+\frac{C_{L_{\dot{\alpha}}} \bar{q} S \bar{c}}{2 V} & 0 & 0 \\
0 & 1 & 0 \\
-\frac{C_{m_{\alpha}} \bar{q} S \bar{c}^{2}}{2 V} & 0 & I_{y y}
\end{array}\right]\left[\begin{array}{c}
\dot{\alpha} \\
\dot{\theta} \\
\dot{q}
\end{array}\right]=\left[\begin{array}{ccc}
m g \gamma-C_{L_{\alpha}} \bar{q} S & -m g \gamma & m V-\frac{C_{L_{q}} \bar{q} S \bar{c}}{2 V} \\
0 & 0 & 1 \\
C_{m_{\alpha}} & 0 & \frac{C_{m_{q}} \bar{q} \bar{c}^{2}}{2 V}
\end{array}\right]\left[\begin{array}{c}
\alpha \\
\theta \\
q
\end{array}\right]+\left[\begin{array}{c}
-C_{L_{\delta_{e}}} \\
0 \\
C_{m_{\delta_{e}}}
\end{array}\right] \delta_{e}
$$

A numerical model for a full-scale generic transport model (GTM) at Mach 0.8 and 30,000 ft with the flight path angle $\gamma=0$ is given by

$$
\left[\begin{array}{c}
\dot{\alpha} \\
\dot{\theta} \\
\dot{q}
\end{array}\right]=\underbrace{\left[\begin{array}{ccc}
-0.7018 & 0 & 0.9761 \\
0 & 0 & 1 \\
-2.6923 & 0 & -0.7322
\end{array}\right]}_{A}\left[\begin{array}{l}
\alpha \\
\theta \\
q
\end{array}\right]+\underbrace{\left[\begin{array}{c}
-0.0573 \\
0 \\
-3.5352
\end{array}\right]}_{B} \delta_{e}
$$

A desired reference model of the pitch attitude is given by

$$
\ddot{\theta}_{m}+2 \zeta \omega_{n} \dot{\theta}_{m}+\omega_{n}^{2} \theta_{m}=\omega_{n}^{2} r
$$

where $\zeta=0.85$ and $\omega_{n}=1.5 \mathrm{rad} / \mathrm{sec}$ are chosen to give a desired handling characteristic.

Let $x=\left[\begin{array}{ccc}\alpha & \theta & q\end{array}\right]^{\top}$ and $u=\delta_{e}$. A nominal controller is designed as $u_{\text {nom }}=-K_{x} x-k_{r} r$ where $K_{x}=$ $\frac{1}{b_{3}}\left[\begin{array}{lll}a_{31} & \omega_{n}^{2} & 2 \zeta \omega_{n}+a_{33}\end{array}\right]=\left[\begin{array}{lll}0.7616 & -0.6365 & -0.5142\end{array}\right]$ and $k_{r}=-\frac{1}{b_{3}} \omega_{n}^{2}=0.6365$. The closed-loop eigenvalues are -0.6582 and $-1.2750 \pm 0.7902 i$. The nominal closed-loop plant is then chosen to be the reference model as

$$
\underbrace{\left[\begin{array}{c}
\dot{\alpha} \\
\dot{\theta} \\
\dot{q}
\end{array}\right]_{m}}_{\dot{x}_{m}}=\underbrace{\left[\begin{array}{ccc}
-0.6582 & -0.0365 & 0.9466 \\
0 & 0 & 1 \\
0 & -2.2500 & -2.5500
\end{array}\right]}_{A_{m}} \underbrace{\left[\begin{array}{c}
\alpha \\
\theta \\
q
\end{array}\right]_{m}}_{x_{m}}+\underbrace{\left[\begin{array}{c}
0.0162 \\
0 \\
1
\end{array}\right]}_{B_{m}} r
$$


Suppose an in-state uncertainty and in-control uncertainty exist in the form of

$$
\Delta B=\left[\begin{array}{c}
0 \\
0 \\
1.7676
\end{array}\right], \Delta A=\underbrace{\left[\begin{array}{c}
-0.0573 \\
0 \\
-1.7676
\end{array}\right]}_{B+\Delta B}\left[\begin{array}{lll}
0 & 0 & -0.2071
\end{array}\right]=\left[\begin{array}{ccc}
0 & 0 & 0.0119 \\
0 & 0 & 0 \\
0 & 0 & 0.3661
\end{array}\right]
$$

which represents a $50 \%$ reduced pitch damping and control effectiveness and $11 \%$ increase in $q$-contribution to $\dot{\alpha}$.

Figures 6 is a plot of estimates of phase and time delay margins from the asymptotic solution computed from Eqs. (75) and (76) as a function of $v$. Note that the phase margin with $\Delta A$ and $\Delta B$ decreases steadily as $v$ increases, while the time delay margin reaches a maximum at about $v=1$. Thus, for practical design purposes, $v$ should be kept between 0 and 1. A large value of $v$ produces a better time delay margin, but also results in a poorer steady-state tracking.

Suppose there exists a $0.02 \mathrm{sec}$ time delay at the input due to a latency in the flight control system. Then from Fig. 6 , a value of $v=0.1$ is selected to provide a time delay margin of about $0.027 \mathrm{sec}$. It should be noted that the time delay margin as computed from Eq. (76) is a conservative estimate corresponding to a large adaptive gain value. So the actual time delay margin should be greater than the estimated value.
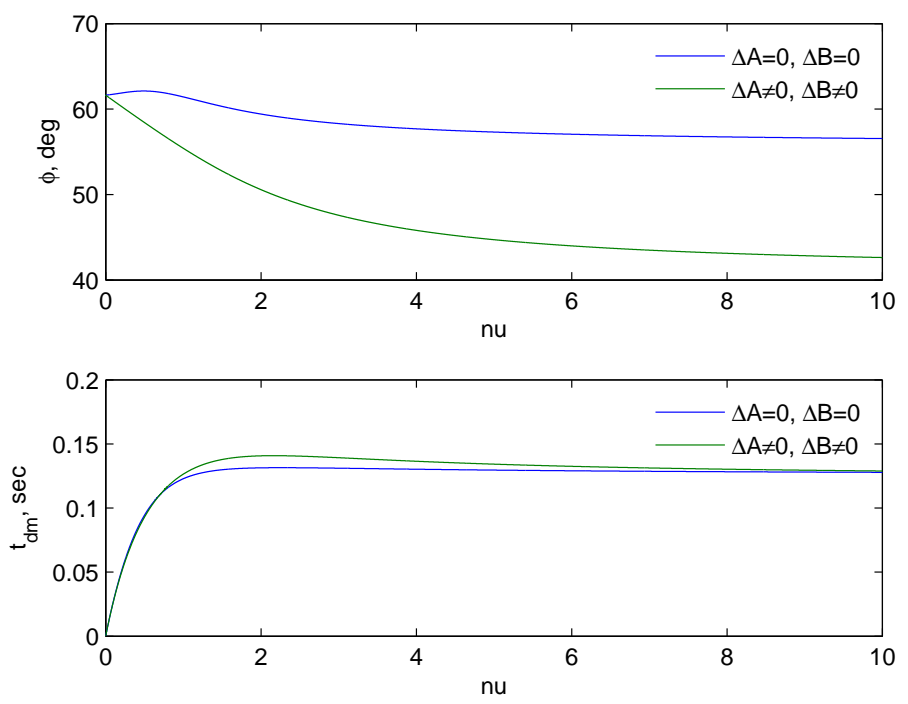

Fig. 6 - Phase and Time Delay Margin Estimates

Figures 7, 8, and 9 show the $\alpha, \theta$, and $q$ responses due to the baseline controller. With no adaptation, the plant is not able to track the reference model very well. For the standard MRAC, an adaptive gain of $\Gamma=1000 I$ is used to enable a tight tracking of the pitch attitude reference signal. The control signal is highly oscillatory during the first 15 sec as evidenced in Fig. 10. The simulation result of the time delay margin of the system with the standard MRAC is observed to be $0.07 \mathrm{sec}$. For the optimal control modification, the design parameter $v=0.1$ is used with the same adaptive gain. The response was more improved without high frequency signals. The observed time delay margin of the optimal control modification adaptive law is $0.3 \mathrm{sec}$ which is significantly greater than that with the standard MRAC. 

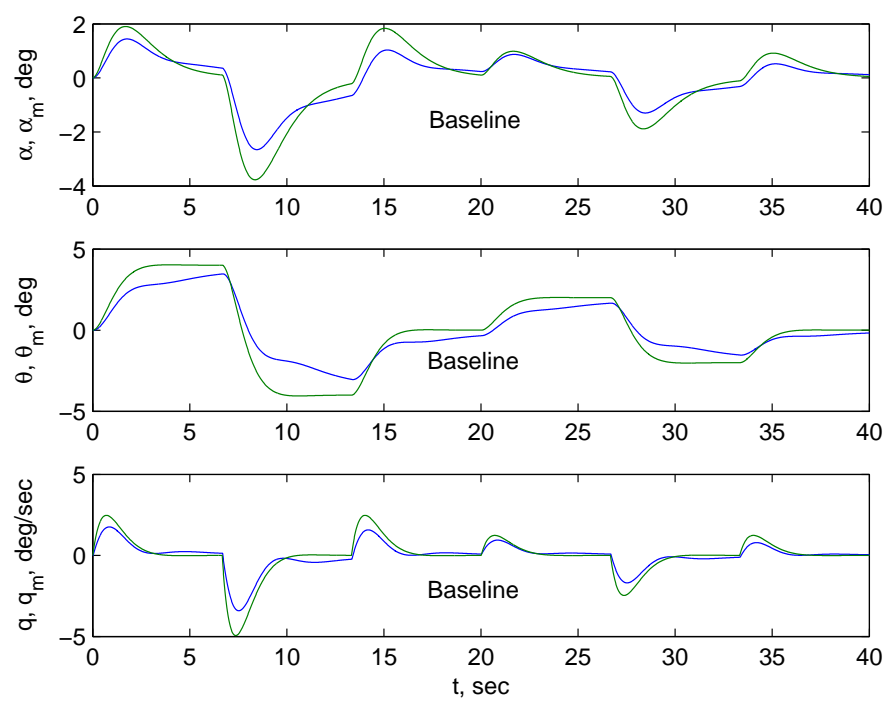

Fig. 7 - $\alpha, \theta$, and $q$ Responses with Baseline Controller
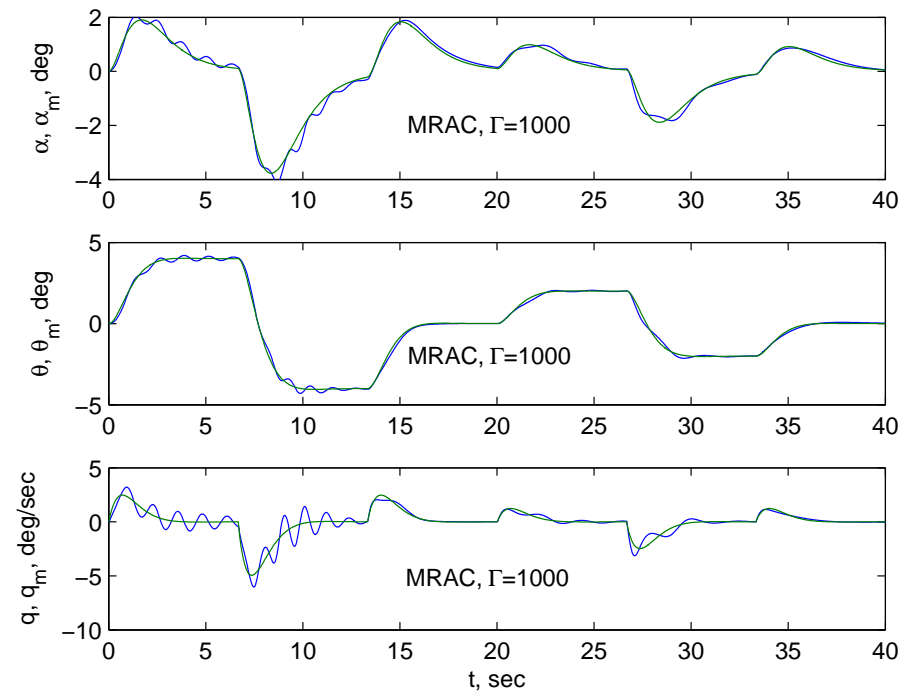

Fig. 8 - $\alpha, \theta$, and $q$ Responses with Standard MRAC 

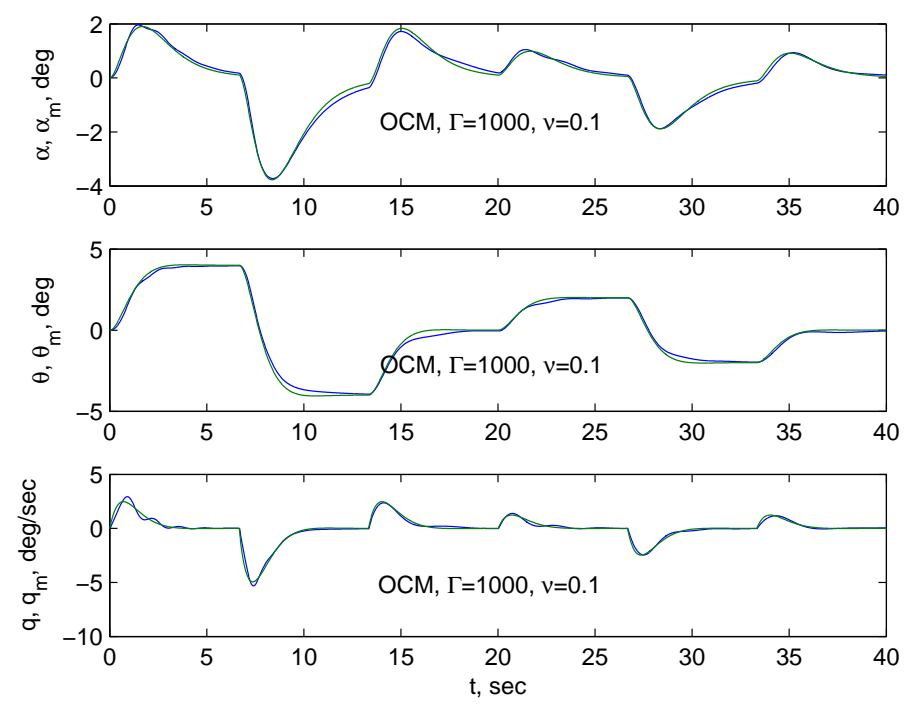

Fig. 9 - $\alpha, \theta$, and $q$ Responses with Optimal Control Modification (OCM)
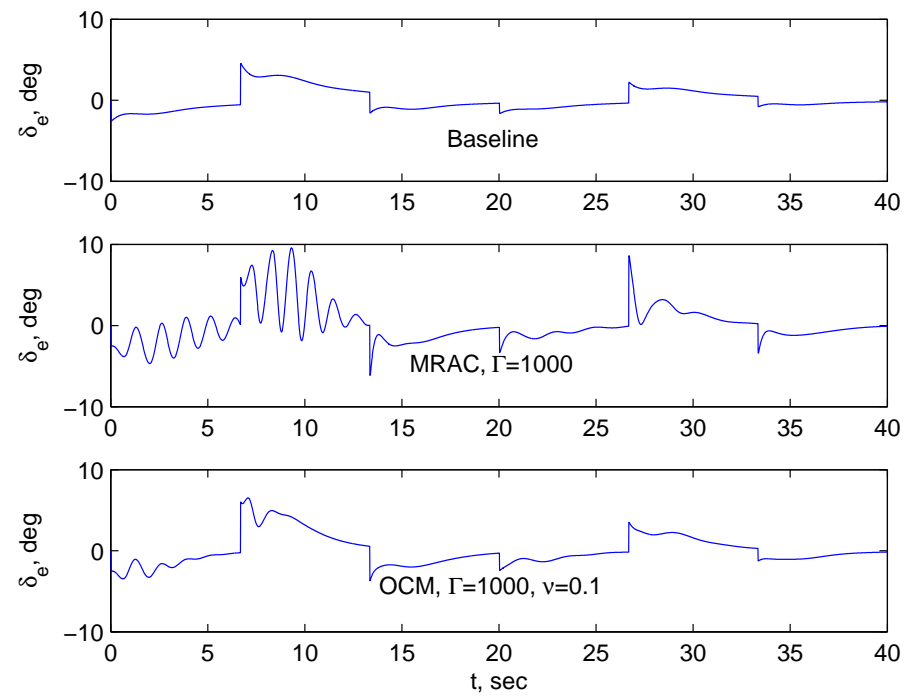

Fig. 10 - Elevator Deflection

\section{Conclusions}

This study shows that the recently developed optimal control modification exhibits a linear asymptotic property for systems with linear matched uncertainty. Using a singular perturbation argument, the outer solution of the adaptive system is governed by an asymptotic linear time invariant system. As a result, the notions of phase and time delay margins for this asymptotic system can be established analytically. With the use of the projection operator, the optimal control modification can be designed to satisfy stability margins in a linear sense by a suitable selection of the free design parameter of the adaptive law. This approach might provide a path toward easing technical challenges with verification and validation of adaptive control systems.

\section{References}

${ }^{1}$ Ioannou, P. and Kokotovic, P., "Instability Analysis and Improvement of Robustness of Adaptive Control," Automatica, Vol. 20, No. 5, 1984, pp. 583-594. 
${ }^{2}$ Narendra, K. S. and Annaswamy, A. M., “A New Adaptive Law for Robust Adaptation Without Persistent Excitation”, IEEE Transactions on Automatic Control, Vol. AC-32, No. 2, pp. 134-145, 1987.

${ }^{3}$ Nguyen, N., Krishnakumar, K., and Boskovic, J., "An Optimal Control Modification to Model-Reference Adaptive Control for Fast Adaptation", AIAA Guidance, Navigation, and Control Conference, AIAA 2008-7283, 2008.

${ }^{4}$ Ioannou, P. A. and Sun, J., Robust Adaptive Control, Prentice-Hall, 1996.

${ }^{5}$ Bryson, A. E. and Ho, Y. C., Applied Optimal Control: Optimization, Estimation, and Control, John Wiley \& Sons Inc., 1979.

${ }^{6}$ Khalil, H. K., Nonlinear Systems, Prentice-Hall, 2002.

${ }^{7}$ Kokotovic, P., Khalil, H., and O'reilly, J., Singular Perturbation Methods in Control: Analysis and Design, Society for Industrial and Applied Mathematics, 1987.

${ }^{8}$ Ardema, M., "Computational Singular Perturbation Method for Dynamical Systems", AIAA Journal of Guidance, Control, and Dynamics, Vol. 14, 661-663, 1981.

${ }^{9}$ Nguyen, N., Ishihara, A., Krishnakumar, K., and Bakhtiari-Nejad, M., "Bounded Linear Stability Analysis - A Time Delay Margin Estimation Approach for Adaptive Control", AIAA Guidance, Navigation, and Control Conference, AIAA-2009-5968, 2009.

${ }^{10}$ Rohrs, C.E., Valavani, L., Athans, M., and Stein, G., "Robustness of Continuous-Time Adaptive Control Algorithms in the Presence of Unmodeled Dynamics", IEEE Transactions on Automatic Control, Vol AC-30, No. 9, pp. 881-889, 1985. 